\title{
Engineering Nucleotide Specificity of Succinyl-CoA Synthetase in Blastocystis: The Emerging Role of Gatekeeper Residues
}

\author{
Kapil Vashisht, ${ }^{\dagger}$ Sonia Verma, ${ }^{\dagger}$ Sunita Gupta, ${ }^{\dagger}$ Andrew M. Lynn, ${ }^{\ddagger}$ Rajnikant Dixit, ${ }^{\dagger}$ Neelima Mishra,
} Neena Valecha, ${ }^{\dagger}$ Karleigh A. Hamblin, ${ }^{\perp, \S}$ Robin Maytum, ${ }^{*}, \|$ Kailash C. Pandey, ${ }^{*},{ }^{\dagger}, @$ and Mark van der Giezen*, ${ }^{\star \perp}$

${ }^{\dagger}$ Host-parasite Interaction Biology Group, National Institute of Malaria Research, ICMR, New Delhi 110077, India

${ }^{\ddagger}$ School of Computational and Integrative Sciences, Jawaharlal Nehru University, New Delhi 110067, India

${ }^{\perp}$ Biosciences, University of Exeter, Stocker Road, Exeter EX4 4QD, U.K.

"School of Life Sciences, University of Bedfordshire, University Square, Luton LU1 3JU, U.K.

Supporting Information
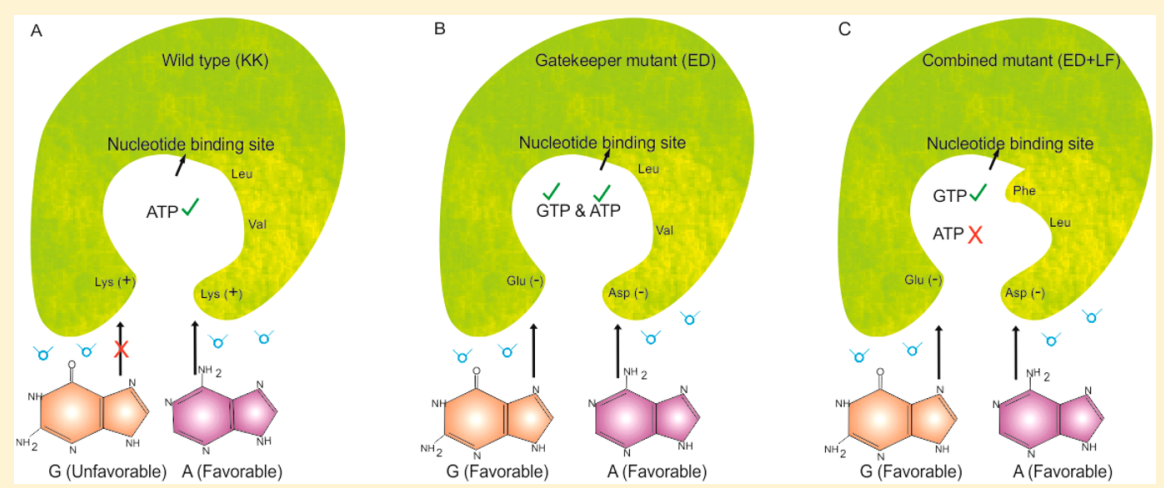

ABSTRACT: Charged, solvent-exposed residues at the entrance to the substrate binding site (gatekeeper residues) produce electrostatic dipole interactions with approaching substrates, and control their access by a novel mechanism called "electrostatic gatekeeper effect". This proof-of-concept study demonstrates that the nucleotide specificity can be engineered by altering the electrostatic properties of the gatekeeper residues outside the binding site. Using Blastocystis succinyl-CoA synthetase (SCS, EC 6.2.1.5), we demonstrated that the gatekeeper mutant (ED) resulted in ATP-specific SCS to show high GTP specificity. Moreover, nucleotide binding site mutant (LF) had no effect on GTP specificity and remained ATP-specific. However, via combination of the gatekeeper mutant with the nucleotide binding site mutant (ED+LF), a complete reversal of nucleotide specificity was obtained with GTP, but no detectable activity was obtained with ATP. This striking result of the combined mutant (ED+LF) was due to two changes; negatively charged gatekeeper residues (ED) favored GTP access, and nucleotide binding site residues (LF) altered ATP binding, which was consistent with the hypothesis of the "electrostatic gatekeeper effect". These results were further supported by molecular modeling and simulation studies. Hence, it is imperative to extend the strategy of the gatekeeper effect in a different range of crucial enzymes (synthetases, kinases, and transferases) to engineer substrate specificity for various industrial applications and substrate-based drug design.

$T^{1}$ he landmark models like "lock and key" and "induced fit" ${ }^{2}$ of enzyme specificity have revolutionized the field of enzymology. Enzyme specificity is a very important characteristic of enzymes and makes them indispensable research tools in the field of biotechnology. There are studies that have been undertaken to engineer coenzyme specificity and redesign secondary structures, ${ }^{3-6}$ and numerous efforts have been made to achieve desired enzyme specificity. From a wider perspective, enzyme specificity is based on molecular interactions between proteins and interacting partners like other proteins, DNA, and ligands. These molecular associations are thought to be driven by interaction free energies arising from structural features like hydrogen bonding and amino acid propensity inside the substrate binding site. ${ }^{7}$ A precise understanding of these interactions requires in-depth analysis of the factors governing these associations. Structural analysis of macromolecules and their interacting partners remains the most promising method for deciphering the rules governing these associations. Molecular recognition of cognate and noncognate ligands by proteins is a well-known occurrence, normally explained by sequence specificity and steric availability inside the binding site, but less understood when proteins have to distinguish

Received: February 5, 2016

Revised: July 27, 2016

Published: August 1, 2016 
between very similar ligands like adenine (A) and guanine $(G)$. Precise determinants of $A / G$ specificity in purine binding proteins are still unclear. To explain the molecular recognition of $A$ and $G$ in nucleotide binding proteins, it was proposed that the distribution of hydrogen bond donors and acceptors from protein atoms and purine rings might be used to differentiate ATP-specific binding sites from GTP-specific binding sites. ${ }^{7}$ The insufficiency of this proposed mechanism in explaining molecular discrimination led Basu et al. to re-examine the question of discrimination between $A$ and $G$ by nucleotide binding proteins. Focusing on the electrostatic potential (ESP) of purine binding sites showed a clear correlation in ESP patterns and $\mathrm{A} / \mathrm{G}$ specificity across protein families. ${ }^{8}$ This study established the role of a strong electrostatic component for molecular discrimination by calculating the ESP of each binding site. Earlier discoveries also showed that individual amino acids do contribute in the overall electrostatic field of a protein that can be calculated by a continuum solvent model. ${ }^{9}$ The electrostatic properties of amino acids in the active site would be of considerable importance as changing the charge of the constituent amino acids in the catalytic site resulted in altered function and overall stability of the protein, ${ }^{9,10}$ but monitoring the effects of changing the electrostatic properties of amino acids in the protein-ligand interactions, at sites near or outside the binding site, still need to be investigated promptly as this is still a gray area of research in the field of enzymology.

Previously, computational analysis using enzyme models of SCS (succinyl-CoA synthetase) from Blastocystis, Escherichia coli, and Sus scorfa (pig) has identified residues interacting with ligands (ATP and GTP). ${ }^{11}$ Strong dipole moments of both ATP and GTP were proposed to be responsible for discriminating nucleotides at the rim surrounding the binding site in Blastocystis SCS. Modeling and unbinding simulations of the complexes with nucleotides showed that GTP flipped to $180^{\circ}$ with a significant decrease in energy, while there was no change in ATP orientation. ${ }^{11}$ An "electrostatic gatekeeper effect" has been hypothesized, which stated that the electrostatic properties of gatekeeper residues influence nucleotide specificity and GTP is restricted from binding to ATP-specific SCS due to this effect.

Being a TCA cycle enzyme, SCS has been studied in detail; its biochemical characterization has been performed, and crystal structures of the enzyme from various organisms have been determined. $^{12-15}$ To the best of our knowledge, there is no experimental evidence available for engineering nucleotide specificity on the basis of electrostatic properties of gatekeeper residues; therefore, our study is a novel and successful attempt to validate the "electrostatic gatekeeper effect". Via a combination of site-directed mutagenesis, enzyme kinetics, modeling, and simulation studies, our results clearly demonstrated that electrostatic dipole interactions control nucleotide access, and additionally, crucial nucleotide binding site residues prevented ATP binding in Blastocystis SCS. Crystal structures of SCS from E. coli (nonspecific, ATP and GTP) and pig (GTPspecific) are available and well-studied. Blastocystis SCS here represents the ATP-specific isoform of the enzyme, and there is not much available information about the molecular mechanism of nucleotide specificity in various isoforms. Therefore, we have chosen Blastocystis SCS (ATP-specific) to validate our "electrostatic gatekeeper effect" and to understand the molecular basis of nucleotide specificity.

Blastocystis is a strict anaerobic human intestinal parasite, which possesses organelles having mitochondrial as well as hydrogenosomal features. ${ }^{16}$ SCS is particularly important in Blastocystis because hydrogenosomes do not have the ability to generate energy through oxidative phosphorylation and SCS generates ATP through substrate-level phosphorylation. ${ }^{11}$ The proposed metabolic pathways of mitochondria like organelles in Blastocystis have been shown to have an incomplete Krebs cycle. Coupling of succinate:succinyl-CoA cycling with acetate formation in Blastocystis has been suggested to conserve the energy of the thioester bond, which is further used in ATP formation. ${ }^{16}$ Because SCS plays a crucial role in energy generation in anaerobic parasites, its role in the life cycle of these parasites is indispensable and it may be targeted in drug discovery. There are other biochemically important enzymes like transferases and kinases from various organisms on which this gatekeeper hypothesis can be tested, and this information would improve our understanding of enzyme specificity of closely related ligands.

\section{EXPERIMENTAL METHODS}

Blastocystis SCS consists of two subunits, $\operatorname{SCS} \alpha$ and $\operatorname{SCS} \beta$. SCS $\alpha$ and SCS $\beta$ genes were separately amplified from Blastocystis hominis strain NandII cDNA using primers listed in Table S1. Both SCS $\alpha$ and SCS $\beta$ subunits were cloned into the pET28a vector (Novagen) separately using appropriate restriction enzymes as mentioned in Table S1. All mutants were generated by using the Q5 site-directed mutagenesis kit (New England Biolabs). A list of gatekeeper residues and their resultant net charges at the surface of the binding site of enzymes are listed in Table S2. All clones containing SCS $\alpha$, wild-type $\operatorname{SCS} \beta$, and different mutants were sequenced to confirm the orientation and open reading frame (ORF) for protein expression with a particular mutation. Positive clones of $\operatorname{SCS} \alpha, \operatorname{SCS} \beta$, and different mutants of Blastocystis were recombinantly produced in a bacterial expression system as described previously. ${ }^{11}$ The SCS $\alpha$ subunits of Blastocystis were expressed as a soluble protein and further purified by Ni-NTA affinity chromatography as described previously. ${ }^{11}$ Pellets for $\operatorname{SCS} \beta$ subunits were processed for isolation of inclusion bodies (IBs), because $\operatorname{SCS} \beta$ subunits of Blastocystis were expressed as IBs. The purification of SCS $\beta$ subunits was performed as described previously. ${ }^{17-19}$ After sodium dodecyl sulfatepolyacrylamide gel electrophoresis (SDS-PAGE) analysis, purified fractions were pooled and concentrated with a 10 $\mathrm{kDa}$ cutoff Centricon (Vivaspin). Buffer exchange was performed with $6 \mathrm{M} \mathrm{Gn}-\mathrm{HCl}$ and $50 \mathrm{mM}$ Tris- $\mathrm{HCl}(\mathrm{pH} \mathrm{8.0})$ until a final volume of $1 \mathrm{~mL}$ was reached.

Different refolding conditions were tried for purified, denatured SCS $\alpha$ and $\operatorname{SCS} \beta$ of Blastocystis as described previously. ${ }^{19-21}$ The maximal activities of enzymes were obtained when $\operatorname{SCS} \alpha$ and $\operatorname{SCS} \beta$ subunits were finally mixed in an equal ratio and refolded by 100 -fold dilution in optimized refolding buffer containing $50 \mathrm{mM}$ Tris- $\mathrm{HCl}, 25 \%$ glycerol, 25 $\mathrm{mM}$ DTT, and $100 \mu \mathrm{M} \mathrm{MgCl} 2$ (pH 7.2). The final protein concentration in refolding buffer was $50 \mu \mathrm{g} / \mathrm{mL}$, and the protein was incubated overnight at $4{ }^{\circ} \mathrm{C}$ while being mildly stirred. Refolded protein was centrifuged at $15000 \mathrm{rpm}$ for 30 min at $4{ }^{\circ} \mathrm{C}$ to remove particulate matter, and the supernatant was concentrated using a $10 \mathrm{kDa}$ cutoff Centricon (Vivaspin).

Circular dichroism experiments were performed on a JASCO-815 spectropolarimeter at the Central Instrumentation Facility of the University of Delhi (South Campus, New Delhi, India). Purified and refolded proteins were exchanged in 10 $\mathrm{mM}$ sodium phosphate buffer $(\mathrm{pH}$ 7.2) and concentrated using 
a $10 \mathrm{kDa}$ cutoff Macrosep (Pall Life Sciences). All experiments were performed in a quartz cell with a path length of $1 \mathrm{~mm}$. Circular dichroism (CD) signals were monitored between 190 and $260 \mathrm{~nm}$ at $25{ }^{\circ} \mathrm{C}$. CD spectra were finally measured by taking the average of the three best scans.

Kinetics of wild-type and mutant enzymes were determined as described previously ${ }^{11}$ with some modifications. The assay buffer consisted of $129 \mu \mathrm{M}$ CoA, $10 \mathrm{mM}$ sodium succinate, 50 $\mathrm{mM} \mathrm{KCl}, 10 \mathrm{mM} \mathrm{MgCl}$, and $50 \mathrm{mM}$ Tris- $\mathrm{HCl}$ ( $\mathrm{pH}$ 7.4). Recombinant wild-type and mutant enzymes were analyzed for kinetic parameters with given concentrations of nucleotides. In each assay of Blastocystis SCS, $30 \mathrm{nM}$ enzyme was used. The reaction was specifically followed by formation of the succinyl$\mathrm{CoA}$ bond at $232 \mathrm{~nm}$. Kinetic parameters were calculated from three independent batches of wild-type and mutant enzymes, using GraphPad Prism 5.

Modeling and Molecular Dynamics Simulations. Model structures of Blastocystis SCS (wild type and mutants), one with GTP and another with ATP as ligands, were generated with Modeler 9v13 using pig SCS [Protein Data Bank (PDB) entry 2FP4 $]^{14}$ and E. coli SCS (PDB entry $1 \mathrm{CQI})^{13}$ structures as templates, respectively. Each model consists of two subunits, $\operatorname{SCS} \alpha$ and $\operatorname{SCS} \beta$, and their respective ligands. The SCS $\alpha$ subunit has the binding site for coenzyme A (CoA), while the $\operatorname{SCS} \beta$ subunit has the nucleotide binding site. Because the pig SCS structure does not have CoA coordinates in it, CoA coordinates were introduced from the E. coli SCS structure into the models with GTP. The best models were selected on the basis of the DOPE score and used for further analysis. Electrostatic surfaces were produced using the EF-surf server and visualized in PDB jviewer. ${ }^{22}$ To observe proteinnucleotide interactions more realistically, we conducted simulations for ATP and GTP starting from the predicted complex models of the wild type, the gatekeeper mutant (ED), the nucleotide binding site mutant (LF), and the combined mutant $(\mathrm{ED}+\mathrm{LF})$. The topologies for ATP and GTP were taken from the AMBER Parameter Database (http://www. pharmacy.manchester.ac.uk/bryce/amber/ $)^{23}$ followed by conversion to GROMACS 5.1.2 ${ }^{24}$ format using an Acpype python script. $^{25}$ The coordinate and topology file of the protein was generated using the pdb2gmx program of the GROMACS package taking parameters from the AMBER99sb-ildn force field and using TIP3P as the water model. All eight complexes of ATP and GTP (wild type and ED, LF, and ED+LF mutants) were preliminarily subjected to energy minimization with a tolerance of $1000 \mathrm{~kJ} \mathrm{~mol}^{-1} \mathrm{~nm}^{-1}$ with the steepest descent method. All bonds were constrained using P-LINCS. After minimization, a short NVT (fixed volume and temperature) MD simulation (200 ps) with positional restraints applied to each system was used to soak the macromolecule into the solvent. A time step of $2 \mathrm{fs}$ was used in all cases, and the systems were coupled to a temperature bath at room temperature using V-rescale, a thermostat that uses velocity rescaling with a stochastic term. Long-range electrostatics was handled using the PME method. Then, two short MD simulations under NPT conditions (fixed temperature and pressure) were performed in which positional restraints were scaled down from 1000 to 100 and from 100 to 0 to facilitate better equilibration of the system. A pressure of 1 bar was coupled using Berendsen's method. Lastly, a production of 15 ns was performed separately for each system with a time step of 2.0 fs and no positional restraints. Trajectories and energy components all were written every 10 ps. Binding free energy calculation methods have emerged as a powerful tool for providing quantitative measures of protein-ligand interactions. In this work, the specificity of a molecule is determined by estimating the binding free energies of ATP and GTP for both wild-type and mutant complexes using molecular mechanics and Poisson-Boltzmann surface area (MM-PBSA) calculations. In MM-PBSA, the binding energy is evaluated according to eq 1 :

$$
\Delta G^{\text {bind }}=\Delta \mathrm{EMM}+\Delta G^{\text {psolv }}+\Delta G^{\text {npsolv }}-T \Delta S
$$

where EMM represents the molecular mechanics contribution expressed as the sum of internal, electrostatics, and van der Waals contributions to binding in vacuo followed by polar $\left(\Delta G^{\text {psolv }}\right)$ and nonpolar $\left(\Delta G^{\text {npsolv }}\right)$ contributions to solvation free energies. The polar solvation energy is calculated by solving the $\mathrm{PB}$ equation, while the nonpolar solvation energy is usually computed by the solvent accessible surface area (SASA) model, the most commonly used nonpolar model. The entropy term $(T \Delta S)$ is excluded from the calculation for relative studies; hence, binding energy will not be comparable to the absolute binding energy. g_mmpbsa was used to calculate binding free energies and also to estimate the energy contribution per residue to the binding energy. The energy components $\Delta \mathrm{EMM}, \Delta G^{\text {psolv }}$, and $\Delta G^{\text {npsolv }}$ of individual atoms were calculated in the bound and unbound form, and subsequently, their contribution to the binding energy of residue $x, \Delta R_{x}$, was calculated. The entropy contribution is not included in $\mathrm{g}$ mmpbsa, ${ }^{26}$ so this binding energy will not be comparable to the absolute binding energy but to the relative binding energy. The root-mean-square deviation (RMSD) calculations were performed for the whole protein backbone, the nucleotide binding domain residue backbone (residues 1251 of $\operatorname{SCS} \beta$ ), the nucleotide interacting residue backbone, and the nucleotides (ATP and GTP). The binding free energies of all eight complexes were calculated by the program g_mmpbsa using molecular dynamics simulation trajectories. All energy components, $\Delta \mathrm{EMM}, \Delta G^{\mathrm{psolv}}$, and $\Delta G^{\text {npsolv }}$, for each complex were calculated every $10 \mathrm{ps}$ from the production trajectory between 10 and $15 \mathrm{~ns}$. g_mmpbsa also provides a script to calculate per-residue decomposition, i.e., the contribution of each residue to binding. In this study, we consider only the $\triangle \mathrm{EMM}$ contribution to binding of each residue, as other values were comparable.

\section{RESULTS}

Mutants Design and Refolded Enzymes. On the basis of homology modeling and unbinding simulations of SCS $\beta$ from Blastocystis, pig, and E. coli, with both nucleotides (ATP and GTP), it was hypothesized that the positively charged residues Lys $^{46}$ and Lys ${ }^{114}$ at the entrance to the binding site act as gatekeepers, which prevent GTP from binding inside ATPspecific Blastocystis SCS. ${ }^{11}$ Hence, these residues were termed gatekeeper residues and are thought to control access of the nucleotide to the binding site. For validation of the "electrostatic gatekeeper effect", we have designed a gatekeeper mutant (ED) by changing Lys ${ }^{46}$ to Glu and Lys ${ }^{114}$ to Asp in Blastocystis, which resulted in negative gatekeeper residues similar to those of pig SCS. Comparison of nucleotide binding sites of SCS isoforms, from Blastocystis and pig revealed two residues, $\mathrm{Val}^{113}$ and $\mathrm{Leu}^{227}$ in Blastocystis SCS, that correspond to Leu ${ }^{113}$ and $\mathrm{Phe}^{227}$ in pig SCS, respectively, and the latter residues were previously suggested to support GTP binding. ${ }^{11}$ Therefore, we designed another nucleotide binding site mutant (LF), 

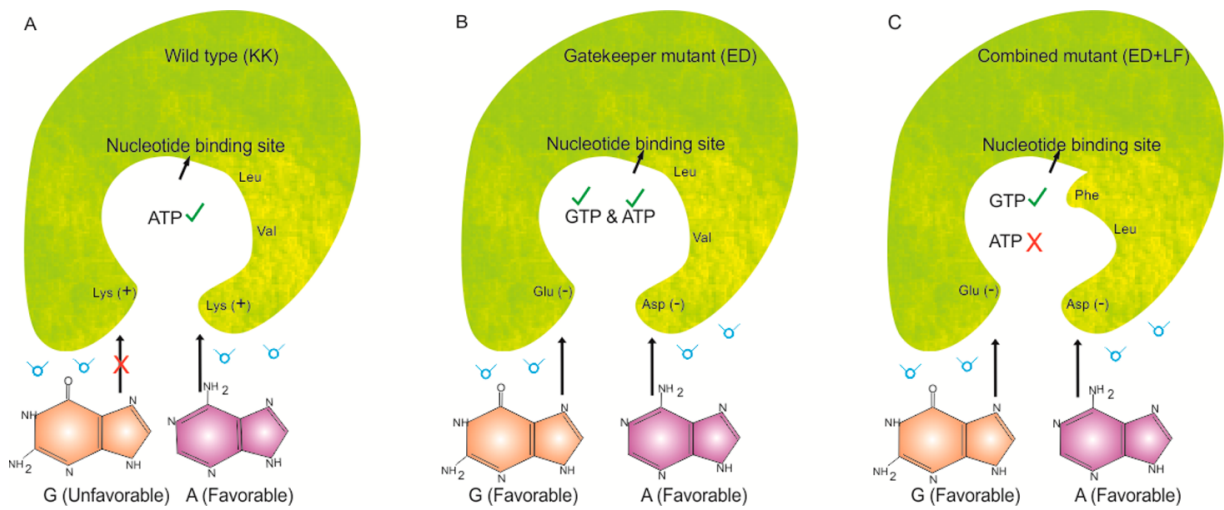

Figure 1. Schematic representation of the "electrostatic gatekeeper effect". (A) The Blastocystis wild-type SCS $\beta$ subunit with positively charged gatekeeper residues (KK) favoring adenine and enzyme is ATP-specific. (B) Gatekeeper mutant (ED) favoring both adenine and guanine with negatively charged gatekeeper residues and showing ATP and GTP specificity. (C) Combined mutant (ED+LF) favoring adenine and guanine with negatively charged gatekeeper residues, but ATP binding hindered due to $\pi-\pi$ stacking interactions with Phe ${ }^{227}$, which therefore resulted in an exclusive GTP-specific enzyme. The water molecules are colored turquoise, and sugar and phosphate groups are not shown because of the similarities.

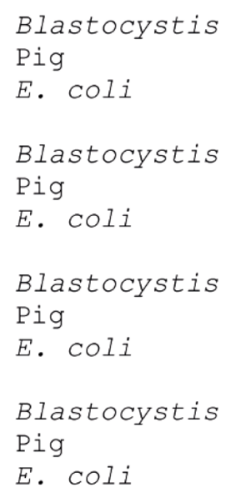

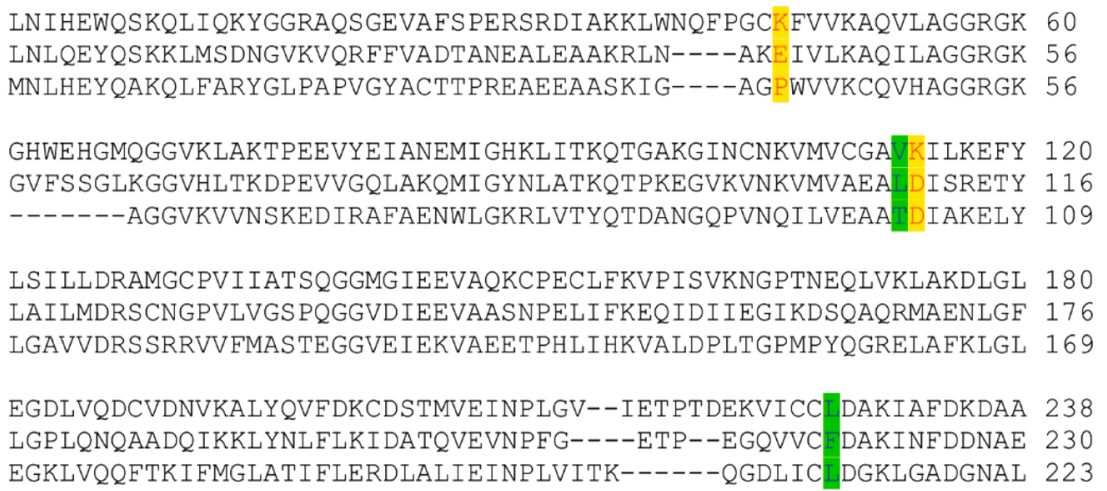

Figure 2. Sequence alignment of SCS $\beta$ subunits of Blastocystis, pig, and E. coli. Gatekeeper residues are highlighted in yellow, and nucleotide binding site residues are highlighted in green. Alignment is done using Clustal W.

mutating $\mathrm{Val}^{113}$ to Leu and $\mathrm{Leu}^{227}$ to Phe, mimicking GTPspecific Pig SCS binding site residues, in side chain contact with the nucleotides. In addition, a combined mutant (ED+LF) was designed by adding both mutations mentioned above, with negatively charged gatekeeper residues $\left(\mathrm{Glu}^{46}\right.$ and $\mathrm{Asp}^{114}$ ) and nucleotide binding site residues ( $\mathrm{Leu}^{113}$ and $\mathrm{Phe}^{227}$ ) that completely mimicked the nucleotide binding site environment of pig SCS. A schematic representation of the "electrostatic gatekeeper effect" shows that positively charged gatekeeper residues (KK) in Blastocystis wild-type $\operatorname{SCS} \beta$ favor adenine, resulting in ATP affinity (Figure 1A). The gatekeeper mutant (ED), with negative gatekeeper residues, allowed both adenine and guanine, resulting in dual nucleotide specificity (Figure $1 B)$. The combined mutant $(E D+L F)$ on the other hand has negative gatekeeper residues and favors both purines but results in only GTP specificity (Figure 1C). The gatekeeper residues and nucleotide binding site residues are indicated in the multiple-sequence alignment of SCS $\beta$ subunits from Blastocystis, pig, and E. coli (Figure 2). The refolded wild-type enzyme and different mutants were concentrated and visualized via SDS-PAGE (Figure 3).

Circular Dichroism Analysis of Wild-Type and Mutant Enzymes. Wild-type SCS and its various mutants were expressed in E. coli, purified, and refolded, and initial velocity experiments were performed to check the activity of enzymes. Evaluation of wild-type SCS and its mutant enzymes by CD

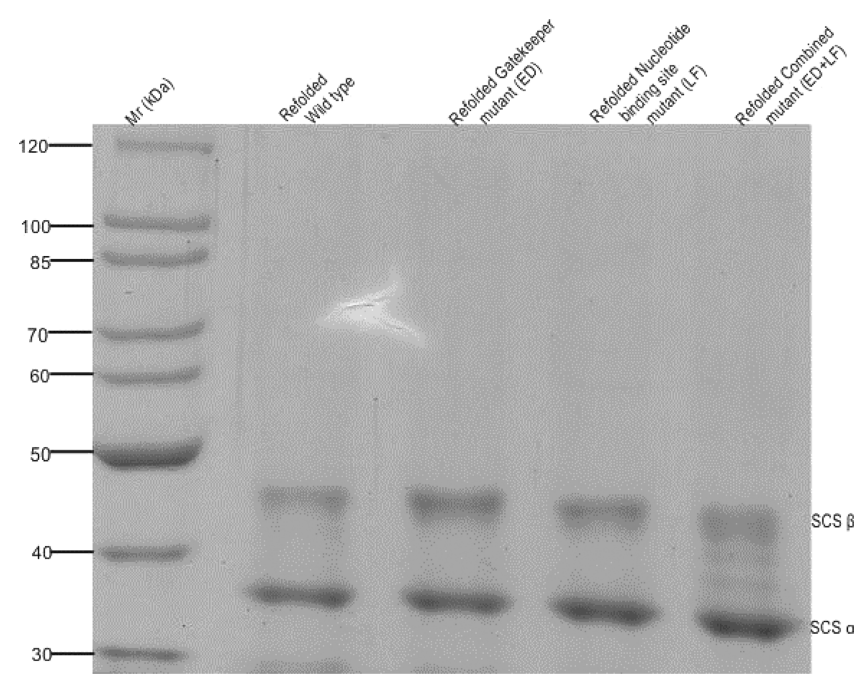

Figure 3. Refolded and purified wild-type and mutant SCS enzymes. SCS $\beta$ and SCS $\alpha$ subunits are shown in the SDS-PAGE gel.

spectra (Figure 4) clearly demonstrated that there were no substantial structural changes in the protein due to mutations in the gatekeeper residues or nucleotide binding site residues. Similar CD spectra for all enzymes showed that mutants also 
Far UV CD spectra

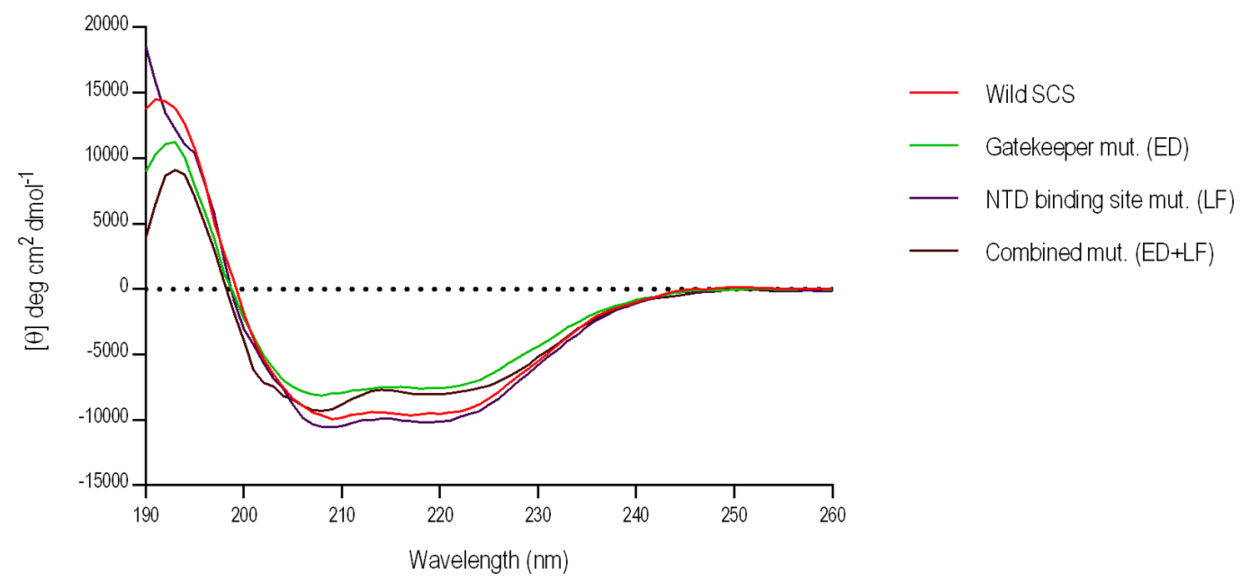

Figure 4. CD spectra of wild-type SCS and its various mutants. The CD spectra (190-260 nm) of the wild type (red), the gatekeeper mutant (ED) (green), the nucleotide binding site mutant (LF) (violet), and the combined mutant (ED+LF) (brown) are shown.

Table 1. Kinetic Parameters of Various Blastocystis Wild-Type and Mutant SCS Enzymes

\begin{tabular}{|c|c|c|c|c|c|c|}
\hline \multirow[b]{2}{*}{ enzyme } & \multicolumn{3}{|c|}{ ATP } & \multicolumn{3}{|c|}{ GTP } \\
\hline & $K_{\mathrm{m}}(\mu \mathrm{M})$ & $k_{\text {cat }}\left(\mathrm{s}^{-1}\right)$ & $k_{\text {cat }} / K_{\mathrm{m}}\left(\mathrm{M}^{-1} \mathrm{~s}^{-1}\right)$ & $K_{\mathrm{m}}(\mu \mathrm{M})$ & $k_{\text {cat }}\left(\mathrm{s}^{-1}\right)$ & $k_{\text {cat }} / K_{\mathrm{m}}\left(\mathrm{M}^{-1} \mathrm{~s}^{-1}\right)$ \\
\hline wild-type SCS & $145 \pm 47$ & $0.0139 \pm 0.002$ & 96 & $\mathrm{ND}^{a}$ & $\mathrm{ND}^{a}$ & $\mathrm{ND}^{a}$ \\
\hline gatekeeper mutant (ED) & $230 \pm 34$ & $0.0176 \pm 0.001$ & 76 & $143 \pm 17$ & $0.0195 \pm 0.001$ & 136 \\
\hline nucleotide binding site mutant (LF) & $265 \pm 50$ & $0.0177 \pm 0.002$ & 66 & $\mathrm{ND}^{a}$ & $\mathrm{ND}^{a}$ & $\mathrm{ND}^{a}$ \\
\hline combined mutant $(\mathrm{ED}+\mathrm{LF})$ & $\mathrm{ND}^{a}$ & $\mathrm{ND}^{a}$ & $\mathrm{ND}^{a}$ & $82 \pm 12$ & $0.0078 \pm 0.0004$ & 96 \\
\hline
\end{tabular}

had a percentage of secondary structure similar to that of wildtype SCS.

Enzyme Kinetics. For kinetic analysis, assay buffers and other experimental conditions were optimized. Kinetic parameters of wild-type and mutant SCS enzymes are summarized in Table 1 . The $K_{\mathrm{m}}$ of wild-type Blastocystis SCS for ATP was $145 \pm 47 \mu \mathrm{M}$, and it showed a $k_{\text {cat }} / K_{\mathrm{m}}$ value of 96 $\mathrm{M}^{-1} \mathrm{~s}^{-1}$ but no detectable activity with GTP (Figure 5A). Strikingly, our gatekeeper mutant enzyme (ED) showed both ATP and GTP specificity but with a clear preference for GTP $\left(K_{\mathrm{m}}=143 \pm 17 \mu \mathrm{M}\right)$ over ATP $\left(K_{\mathrm{m}}=230 \pm 34 \mu \mathrm{M}\right)$ (Figure $5 \mathrm{~B})$. The catalytic efficiency of the gatekeeper mutant for GTP was $136 \mathrm{M}^{-1} \mathrm{~s}^{-1}$, compared to a value of $76 \mathrm{M}^{-1} \mathrm{~s}^{-1}$ for ATP, showing an approximate 2 -fold increase with GTP. The nucleotide binding site mutant (LF) showed a clear preference for ATP as in the wild type, but with a lower affinity with a $K_{\mathrm{m}}$ of $265 \pm 50 \mu \mathrm{M}$ (Figure 5C). Strikingly, in the combined mutant, we observed a complete reversal of nucleotide specificity. Kinetic analysis of the combined mutant showed a high affinity for GTP; however, no major detectable ATP activity was observed (up to $1 \mathrm{mM}$ ), and the $K_{\mathrm{m}}$ value for GTP was $82 \pm 12 \mu \mathrm{M}$. The $k_{\text {cat }} / K_{\mathrm{m}}$ value of the combined mutant was $96 \mathrm{M}^{-1} \mathrm{~s}^{-1}$, which showed that it had a catalytic efficiency with GTP similar to that of the ATP-specific wild-type enzyme (Figure 5D). These results supported the hypothesis that negatively charged gatekeeper residues preferred GTP as in the case of pig SCS, while the nucleotide binding site mutant had no effect on GTP binding, suggesting that gatekeepers did control nucleotide access. In addition, the strongest effect was observed when the electrostatic properties of the gatekeeper residues were altered and the crucial nucleotide binding site residues were modified, which resulted in complete reversal of nucleotide specificity.
Molecular Modeling and Simulation Analysis of Blastocystis SCS and Its Mutants. To investigate the molecular basis of nucleotide specificity in Blastocystis SCS, homology models were constructed using X-ray structures of SCS $\beta$ subunits from pig and E. coli SCS templates. Electrostatic surface calculations performed on these models showed that wild-type Blastocystis SCS $\beta$ (Figure 6A) and the nucleotide binding site mutant (LF) (Figure $6 \mathrm{~B}$ ) both had a net positive surface at the binding site entrance, while the gatekeeper mutant (ED) (Figure 6C) and combined mutant (ED+LF) (Figure 6D) both had net negative surfaces at the binding site entrance. For reference, electrostatic surface charge models showed a net negative charge at the entrance to the binding site in pig SCS $\beta$ (Figure 6E), while E. coli $\operatorname{SCS} \beta$ (Figure 6F) showed a neutral surface at the binding site. The binding free energies of all eight complexes were calculated by the program g_mmpbsa using equilibrated molecular dynamics simulation trajectories to sample ensemble conformers. Although it did not include the entropy parameter and hence could not be used to calculate the absolute binding free energy, $g$ mmpbsa can be used to estimate the relative binding free energy between ranges of interacting moieties as well as provide a decomposition of the residue-wise contribution to binding. The overall calculated $\Delta G$ shows a good correlation with our experimental results as shown in Table S3, along with the electrostatic, van der Waals, and solvation energy components of all the complexes. The $\Delta G$ for GTP in the case of the gatekeeper mutant (ED) and the combined mutant $(\mathrm{ED}+\mathrm{LF})$ is significantly larger than that of ATP, and this distinction is largely from the difference in the contribution of the electrostatic component of the calculation. A further examination of the per-residue contribution to binding (Table S4) from the same calculation showed that $\mathrm{Arg}^{58}$, part of the conserved 
A

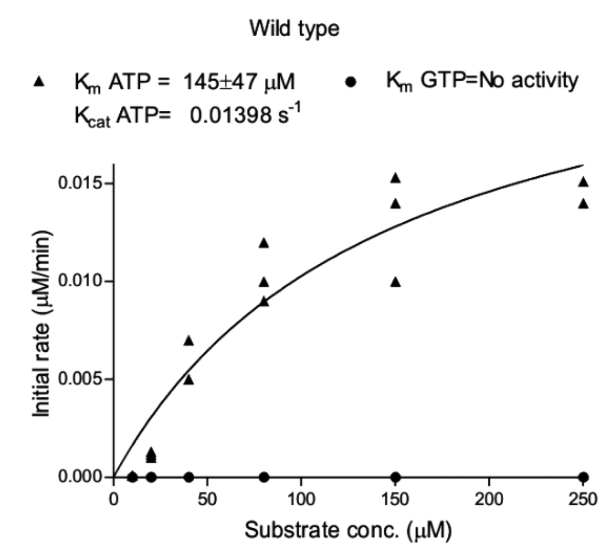

C

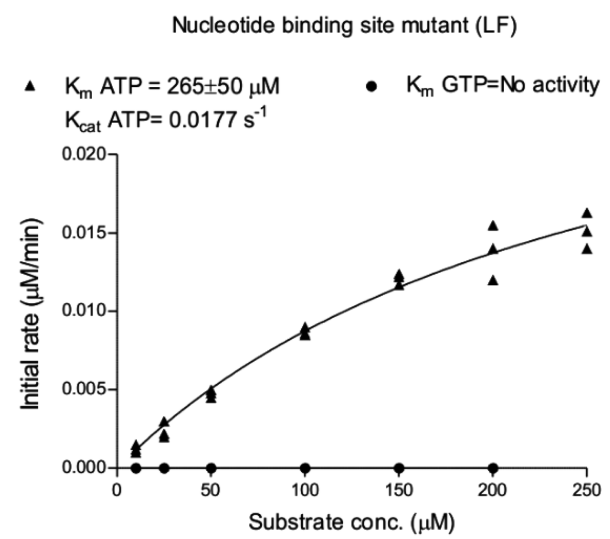

B

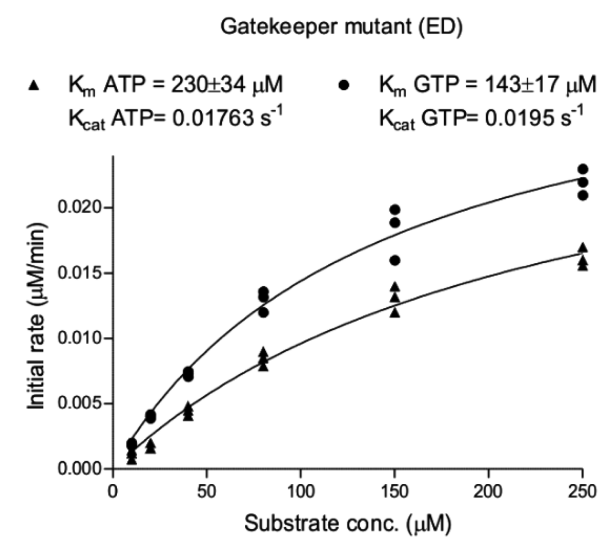

D

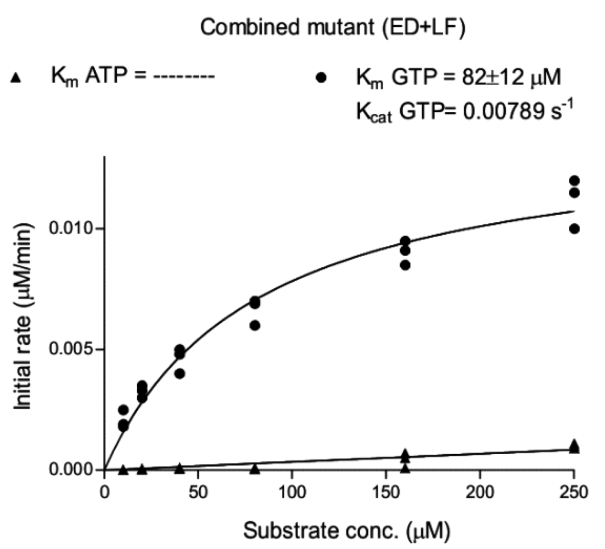

Figure 5. Enzyme kinetics of Blastocystis SCS. Michaelis-Menten plots for kinetic measurements of Blastocystis SCS with variable concentrations of ATP and GTP. Graphs show the initial rate vs ATP and GTP concentration: (A) wild-type SCS, (B) gatekeeper mutant (ED), (C) nucleotide binding site mutant (LF), and (D) combined mutant (ED+LF). In panel C, the LF mutant has a $K_{\mathrm{m}}$ higher than the highest substrate concentration used in the experiment. Replicate values for ATP and GTP are indicated in each graph from three different assays.

GRG motif, ${ }^{14}$ makes a major contribution to the interaction with the phosphate anion. The charged residues interacting with the phosphate anion. The charged residues interacting with the phosphate and nucleotide binding site residues from wild-type and mutants are highlighted in Figure 7, which shows representative conformers used to calculate the binding energy.

\section{DISCUSSION}

SCS was discovered in the 1950s, and X-ray structures of complexes with ATP and GTP have been published; however, the mechanism of its nucleotide specificity is still not clear. Traditional models of enzyme recognition suggest that the process of catalysis is only the function of geometric and electrostatic complementarity between the active site and substrate. Here, we have demonstrated that GTP is restricted from binding to ATP-specific Blastocystis SCS by positively charged gatekeeper residues. In this study, we have successfully reversed the nucleotide specificity of ATP-specific SCS to exclusively GTP-specific SCS. Computational analysis of nucleotide binding sites from Blastocystis and pig SCS $\beta$ has identified 17 residues in close contact with the nucleotides. ${ }^{11}$ The electrostatic surface charge distributions of Blastocystis, E. coli, and pig SCS (Figure 6) have clearly demonstrated the effect of gatekeeper residues on the surface charge, which as a result affected the specificity of the corresponding substrates, i.e., ATP and GTP. Previous studies have reported that pig SCS $(\text { GTP-specific })^{14}$ has negative gatekeeper residues (ED) while Blastocystis SCS (ATP-specific) ${ }^{11}$ has positive gatekeeper residues (KK). The gatekeeper residues (PD) of E. coli (nonspecific) $)^{13}$ allowed SCS to bind to both substrates but showed a higher affinity for GTP. Therefore, charged gatekeeper residues were thought to control access of the nucleotide to the binding site, and thus, we hypothesized that changing the charge of gatekeeper residues would affect nucleotide specificity. The high GTP affinity in the gatekeeper mutant (ED) of ATP-specific SCS proved that negatively charged gatekeeper residues, as in pig SCS allowed access to GTP in addition to ATP, while all other binding site residues remained conserved. ${ }^{11}$ Both nucleotides possess a strong dipole moment; however, GTP has a second dipole, approximately orthogonal to the first, pointing from the carbonyl oxygen at $\mathrm{C}$ 6 to the amino group at C-2. ${ }^{11}$ The dipole moments of adenine and guanine are 2.55 and $6.98 \mathrm{D}$, respectively. ${ }^{27}$ The nucleotide binding site mutant (LF) demonstrated ATP specificity, although with a lower affinity, even with GTP supporting Leu and Phe residues inside the binding site as suggested previously for pig SCS $\beta .^{11}$ This result clearly showed that GTP did not have access due to positively charged gatekeeper residues (KK) in the nucleotide binding site mutant (LF). The combined mutant $(\mathrm{ED}+\mathrm{LF})$ demonstrated a complete reversal of 


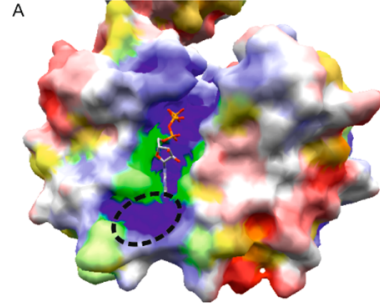

Blastocystis SCS $\beta$

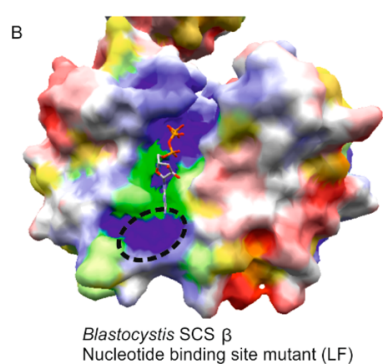

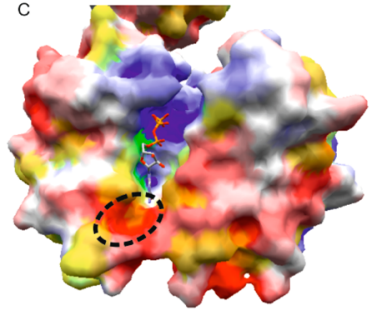

Blastocystis SCS $\beta$ Gatekeeper mutant (ED)

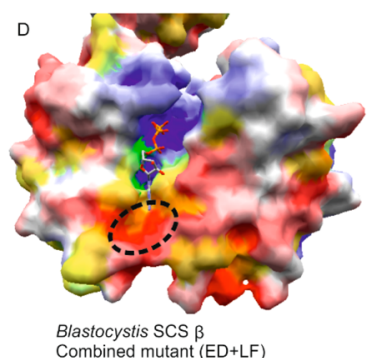

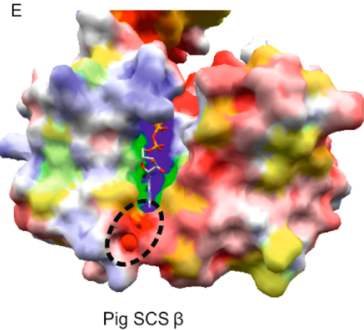

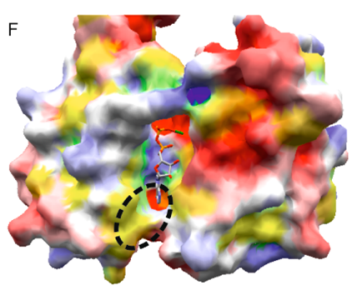

E. colis SCS $\beta$

Figure 6. Electrostatic surface models of the SCS $\beta$ nucleotide binding region. Electrostatic surfaces of the gatekeeper region of SCS $\beta$ are indicated with black ovals. Gatekeeper region in (A) Blastocystis wild-type SCS, (B) nucleotide binding site mutant (LF), (C) gatekeeper mutant (ED), and (D) combined mutant (ED+LF) showing the effect of the change in charge in the gatekeeper region. (E) The negatively charged gatekeeper region in pig SCS and (F) the neutral gatekeeper region in E. coli SCS are shown. Red electrostatic surfaces indicate overall negative gatekeeper residues, whereas blue electrostatic surfaces indicate positive gatekeeper residues. The electrostatic surfaces were prepared by using Modeller9 V1032 and efsurf server and visualized in PDBj viewer.

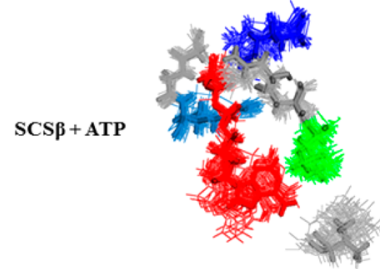

Wild type

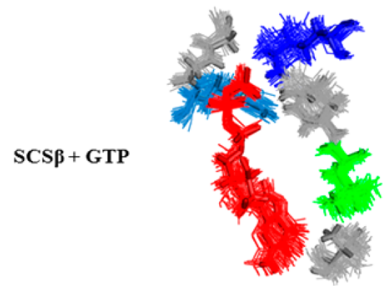

Wild type

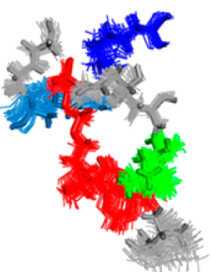

Gatekeeper mutant (ED)

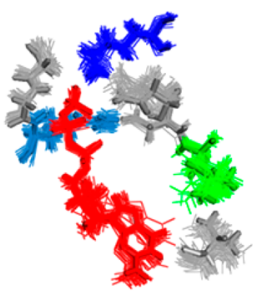

Gatekeeper mutant (ED)

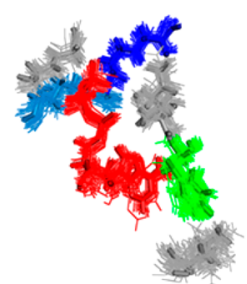

Nucleotide binding site mutant (LF)

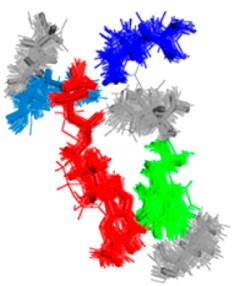

Nucleotide binding site mutant (LF)

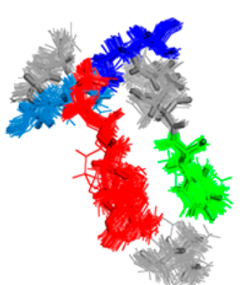

Combined mutant (ED+LF)

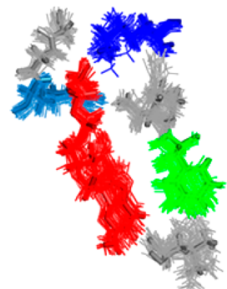

Combined mutant (ED+LF)

Figure 7. Snapshots of molecular dynamic simulations (frames) for the Blastocystis SCS nucleotide binding site with ATP and GTP. Figures show ATP and GTP (red color) inside the nucleotide binding site of SCS with Leu ${ }^{227}$ (green), Lys $^{230}$ (blue), and Arg ${ }^{58}$ (sky blue) in all the systems. The nucleotide binding site mutant (LF) and the combined mutant (ED+LF) have Phe ${ }^{227}$ (green) in place of Leu ${ }^{227}$.

nucleotide specificity with exclusive GTP specificity. Computational studies based on binding energy suggest that GTP has a higher affinity in both the combined mutant and the gatekeeper mutant. While this is confirmed with the gatekeeper mutant, ATP hydrolysis is completely abrogated in the combined mutant. Although the binding energy suggests that GTP has an enhanced electrostatic interaction in these mutants, we speculate that the role of phenylalanine is to lock ATP through $\pi-\pi$ interactions in a conformation unfavorable for hydrolysis. $^{28}$ This may also explain our observation with enzyme kinetic results in which the LF mutant has a reduced ATP affinity $\left(K_{\mathrm{m}}=265 \pm 50 \mu \mathrm{M}\right)$ compared to that of wild-type SCS $\left(K_{\mathrm{m}}=145 \pm 47 \mu \mathrm{M}\right)$. However, these calculations and their interpretation might be influenced by the initial pose and ensemble sampling and would require more extensive study prior to confirmation. Both wild-type ATP-specific SCS and the nucleotide binding site mutant (LF) showed the capability of binding GTP with high affinity, if it was available inside the binding site. However, this possibility was excluded because of the gatekeeper residues present in the enzymes mentioned above, which prevented access of GTP to the binding site. The results presented above suggested that SCS controlled the nucleotide access via the electrostatic properties of the gatekeeper residues. This proposed nucleotide specificity mechanism allows independent evolution of the residues determining the catalysis and selectivity of the enzyme. Hence, mutations outside the binding site of a "generalist" 
enzyme can evolve toward a "specialist" function with minimal perturbation of the binding site residues.

There are reports available in which gatekeeper residues have been explored for discrimination and engineering substrate specificity other than SCS. In aminoglycoside 2"-phosphotransferases (APHs), nucleotide specificity is controlled by a bulky Tyr gatekeeper residue. In exclusive GTP-specific isoforms, Tyr blocks entry of ATP; thus, adding the Tyr gatekeeper residue to ATP-specific isoforms resulted in a high GTP affinity. ${ }^{29}$ Computational analysis of DNA polymerase $\mu$ with its cognate and noncognate ligands has suggested the role of gatekeeper residues in tightening the nucleotide binding pocket, which in turn alters the electrostatic potential, in addition to active site distortion by crucial residues. ${ }^{30}$ In $\beta$ lactamase $(\mathrm{BlaC}), \mathrm{Ile}^{105}$ acted as a gatekeeper residue controlling access of the substrate to active site. Mutation of a gatekeeper residue $\left(\mathrm{I}^{105} \mathrm{~F}\right)$ was thought to open up a space for increased antibiotic resistance and enhanced catalytic efficiency. ${ }^{31}$ In addition, in the case of tyrosine kinases, the inactive enzyme can be activated by mutating a gatekeeper residue (threonine) at the active site and the hydrophobic spine can be created by enzyme engineering, which causes kinase inactivation. $^{32}$ Cofactor switching has also been shown to be important in ketol-acid reductoisomerases (KARIs), as NADHdependent enzymes have enhanced catalytic efficiency compared to that of the wild-type enzyme with NADPH. ${ }^{33}$

Unlike the studies mentioned above, our gatekeeper concept suggests that SCS appears to retain a binding pocket that is capable of binding either substrate or evolved as an alternate mechanism of specificity by changing key residues (charged gatekeeper residues) controlling access to the binding site of the enzyme. In this study, our results have demonstrated that charged gatekeeper residues control the access to the nucleotide binding site of SCS and substrate specificity could be engineered by altering the electrostatic properties of the gatekeeper residues, and further nucleotide binding site modifications were necessary for complete reversal of nucleotide specificity. This gatekeeper hypothesis validated a new role of gatekeeper residues in molecular recognition of ligands on the basis of electrostatic properties. This study emphasized the emerging role of gatekeeper residues in switching the substrate specificity of SCS. This finding has implications for the molecular evolution of enzymes as well as for structure-based drug design and modification of substrate specificity of various enzymes for use in various industrial applications.

\section{ASSOCIATED CONTENT}

\section{S Supporting Information}

The Supporting Information is available free of charge on the ACS Publications website at DOI: 10.1021/acs.biochem.6b00098.

List of primers used for cloning of $\operatorname{SCS} \alpha$ and $\operatorname{SCS} \beta$ subunits from Blastocystis (Table S1), list of gatekeeper residues and their resultant mutants with net charges at the surface of the binding site of Blastocystis SCS (Table S2), different energy values for each system (Table S3), per-residue decomposition of all the binding site residues in SCS $\beta$ subunits (Table S4), G_Cluster results (Table S5), and RMSD plots of various moieties during simulation trajectories (Figure S1) (PDF)

\section{AUTHOR INFORMATION}

\section{Corresponding Authors}

*Phone: 91-7552533106. E-mail: kailash.pandey.nireh@gov.in.

*Phone: 44-1582489264. E-mail: robin.maytum@beds.ac.uk.

*Phone: 44-1392723483. E-mail: m.vandergiezen@exeter.ac.uk.

\section{Present Addresses}

${ }^{\S}$ K.A.H.: CBR division, Defence Science and Technology Laboratory, Porton Down, Salisbury, Wiltshire SP4 0JQ U.K.

${ }^{\circledR}$ K.C.P.: Department of Biochemistry, National Institute for Research in Environmental Health, Indian Council of Medical Research, Bhopal 462001, India.

\section{Author Contributions}

K.C.P., K.V., S.V., S.G., A.M.L., K.A.H., R.M. and M.v.d.G. designed the study and performed experiments, analyzed the data, and wrote the paper. R.D., N.M., and N.V. analyzed the data and wrote the paper. All authors approved the final version of the manuscript.

\section{Funding}

Work is supported by the National Institute of Malaria Research, Indian Council of Medical Research, New Delhi and Dept. of Biotechnology, New Delhi. K.C.P. is a recipient of the Prof. Ramalingaswami Fellowship (Department of Biotechnology, Government of India (BT/HRD/35/02/2006), K.V. is a recipient of UGC Senior Research Fellowship, M.v.d.G. is grateful for support from the University of Exeter and the Wellcome Trust (078566/A/05/Z).

\section{Notes}

The authors declare no competing financial interest.

\section{ACKNOWLEDGMENTS}

We thank Dr. C. Rune Stensvold (Statens Serum Institute, Copenhagen, Denmark) for his generous gift of Blastocystis culture. We also acknowledge Goa University for academic support for the Ph.D program of K.V. This technique has been submitted for patent at ICMR, and the patent application number is $3928 / / \mathrm{DEL} / 2014$. This paper bears the NIMR publication screening committee approval number $037 / 2015$.

\section{REFERENCES}

(1) Fischer, E. (1894) Einfluss der Configuration auf die Wirkung der Enzyme. Ber. Dtsch. Chem. Ges. 27, 2985-2993.

(2) Koshland, D. E. (1958) Application of a Theory of Enzyme Specificity to Protein Synthesis. Proc. Natl. Acad. Sci. U. S. A. 44, 98104.

(3) Scrutton, N. S., Berry, A., and Perham, R. N. (1990) Redesign of the coenzyme specificity of a dehydrogenase by protein engineering. Nature 343, 38-43.

(4) Chen, R, Greer, a, and Dean, a M. (1996) Redesigning secondary structure to invert coenzyme specificity in isopropylmalate dehydrogenase. Proc. Natl. Acad. Sci. U. S. A. 93, 12171-12176.

(5) Zhang, L., Wang, L., Kao, Y.-T., Qiu, W., Yang, Y., Okobiah, O., and Zhong, D. (2007) Mapping hydration dynamics around a protein surface. Proc. Natl. Acad. Sci. U. S. A. 104, 18461-18466.

(6) Tracewell, C. a, and Arnold, F. H. (2009) Directed enzyme evolution: climbing fitness peaks one amino acid at a time. Curr. Opin. Chem. Biol. 13, 3-9.

(7) Nobeli, I., Laskowski, R. a, Valdar, W. S., and Thornton, J. M. (2001) On the molecular discrimination between adenine and guanine by proteins. Nucleic Acids Res. 29, 4294-4309.

(8) Basu, G., Sivanesan, D., Kawabata, T., and Go, N. (2004) Electrostatic potential of nucleotide-free protein is sufficient for discrimination between adenine and guanine-specific binding sites. J. Mol. Biol. 342, 1053-1066. 
(9) Sternberg, M. J. E., Hayes, F. R. F., Russell, A. J., Thomas, P. G., and Fersht, A. R. (1987) Prediction of electrostatic effects of engineering of protein charges. Nature 330, 86-88.

(10) Gilson, M. K., and Honig, B. H. (1987) Calculation of electrostatic potentials in an enzyme active site. Nature 330, 84-86.

(11) Hamblin, K., Standley, D. M., Rogers, M. B., Stechmann, A., Roger, A. J., Maytum, R., and van der Giezen, M. (2008) Localization and nucleotide specificity of Blastocystis succinyl-CoA synthetase. Mol. Microbiol. 68, 1395-1405.

(12) Bridger, W. A., Wolodko, W. T., Henning, W., Upton, C., Majumdar, R., and Williams, S. P. (1987) The subunits of succinylcoenzyme A synthetase-function and assembly. Biochem. Soc. Symp. 54, 103-111.

(13) Wolodko, W. T., Fraser, M. E., James, M. N. G., and Bridger, W. A. (1994) The crystal structure of succinyl-CoA synthetase from Escherichia. J. Biol. Chem. 269, 10883-10890.

(14) Fraser, M. E., James, M. N., Bridger, W. a, and Wolodko, W. T. (2000) Phosphorylated and dephosphorylated structures of pig heart, GTP-specific succinyl-CoA synthetase. J. Mol. Biol. 299, 1325-1339.

(15) Fraser, M. E., Hayakawa, K., Hume, M. S., Ryan, D. G., and Brownie, E. R. (2006) Interactions of GTP with the ATP-grasp domain of GTP-specific succinyl-CoA synthetase. J. Biol. Chem. 281, 11058-11065.

(16) Stechmann, A., Hamblin, K., Perez-Brocal, V., Gaston, D., Richmond, G. S., van der Giezen, M., Clark, C. G., and Roger, A. J. (2008) Organelles in Blastocystis that blur the distinction between mitochondria and hydrogenosomes. Curr. Biol. 18, 580-585.

(17) Pandey, K. C., Singh, N., Arastu-kapur, S., Bogyo, M., and Rosenthal, P. J. (2006) Falstatin, a cysteine protease inhibitor of Plasmodium falciparum, facilitates erythrocyte invasion. PLoS Pathog. 2, e117.

(18) Pandey, K. C., Wang, S. X., Sijwali, P. S., Lau, A. L., McKerrow, J. H., and Rosenthal, P. J. (2005) The Plasmodium falciparum cysteine protease falcipain-2 captures its substrate, hemoglobin, via a unique motif. Proc. Natl. Acad. Sci. U. S. A. 102, 9138-9143.

(19) Pearson, P. H., and Bridger, W. A. (1975) Isolation of the alpha and beta subunits of Escherichia coli succinyl coenzyme A synthetase and their recombination into active enzyme. J. Biol. Chem. 250, 44514455.

(20) Nishimura, J. S., Ybarra, J., Mitchell, T., and Horowitz, P. M. (1988) Isolation, amino acid analyses and refolding of subunits of pig heart succinyl-CoA synthetase. Biochem. J. 250, 429-434.

(21) Fraser, M. E., Joyce, M. A., Ryan, D. G., and Wolodko, W. T. (2002) Two glutamate residues, Glu 208 alpha and Glu 197 beta, are crucial for phosphorylation and dephosphorylation of the active-site histidine residue in succinyl-CoA synthetase. Biochemistry 41, 537546.

(22) Kinoshita, K., and Nakamura, H. (2004) eF-site and PDBjViewer: database and viewer for protein functional sites. Bioinformatics 20, 1329-1330.

(23) Wang, J., Wolf, R. M., Caldwell, J. W., Kollman, P. a., and Case, D. a. (2004) Development and testing of a general Amber force field. J. Comput. Chem. 25, 1157-1174.

(24) Van Der Spoel, D., Lindahl, E., Hess, B., Groenhof, G., Mark, A. E., and Berendsen, H. J. C. (2005) GROMACS: Fast, flexible, and free. J. Comput. Chem. 26, 1701-1718.

(25) Sousa da Silva, A. W., and Vranken, W. F. (2012) ACPYPE AnteChamber PYthon Parser interfacE. BMC Res. Notes 5, 367.

(26) Kumari, R, Kumar, R, and Lynn, A. (2014) g-mmpbsa- A GROMACS tool for high-throughput MM-PBSA calculations. J. Chem. Inf. Model. 54, 1951-1962.

(27) Kilina, S., Tretiak, S., Yarotski, D. A., Zhu, J., Modine, N., Taylor, A., and Balatsky, A. V. (2007) Electronic Properties of DNA Base Molecules Adsorbed on a Metallic Surface. J. Phys. Chem. C 111, 14541-14551.

(28) Hofbauerová, K., Kopecký, V., Ettrich, R., Kubala, M., Teisinger, J., and Amler, E. (2003) ATP-binding is stabilized by a stacking interaction within the binding site of $\mathrm{Na}+/ \mathrm{K}+$-ATPase. Biochem. Biophys. Res. Commun. 306, 416-420.
(29) Bhattacharya, M., Toth, M., Smith, C. a, and Vakulenko, S. B. (2013) Bulky "gatekeeper" residue changes the cosubstrate specificity of aminoglycoside 2"'-phosphotransferase IIa. Antimicrob. Agents Chemother. 57, 3763-3766.

(30) Li, X., Wu, F., and Beard, D. a. (2012) Identification of the kinetic mechanism of succinyl-CoA synthetase. Biosci. Rep. 33, 145163.

(31) Feiler, C., Fisher, A. C., Boock, J. T., Marrichi, M. J., Wright, L., Schmidpeter, P. a M., Blankenfeldt, W., Pavelka, M., and DeLisa, M. P. (2013) Directed evolution of Mycobacterium tuberculosis $\beta$-lactamase reveals gatekeeper residue that regulates antibiotic resistance and catalytic efficiency. PLoS One 8, e73123.

(32) Azam, M., Seeliger, M. A., Gray, N. S., Kuriyan, J., and Daley, G. Q. (2008) Activation of tyrosine kinases by mutation of the gatekeeper threonine. Nat. Struct. Mol. Biol. 15, 1109-1118.

(33) Brinkmann-Chen, S., Flock, T., Cahn, J. K. B., Snow, C. D., Brustad, E. M., McIntosh, J. a, Meinhold, P., Zhang, L., and Arnold, F. H. (2013) General approach to reversing ketol-acid reductoisomerase cofactor dependence from NADPH to NADH. Proc. Natl. Acad. Sci. U. S. A. $110,10946-51$. 